Article

\title{
A Quantum-Like Model of Information Processing in the Brain
}

\author{
Andrei Khrennikov ${ }^{1, *}$ and Masanari Asano ${ }^{2}$ \\ 1 International Center for Mathematical Modeling in Physics and Cognitive Sciences, Mathematical Institute, \\ Linnaeus University, SE-351 95 Växjö , Sweden \\ 2 Liberal Arts Division, National Institute of Technology, Tokuyama College, Gakuendai, Shunan, \\ Yamaguchi 745-8585, Japan; asano@tokuyama.ac.jp \\ * Correspondence: Andrei.Khrennikov@lnu.se; Tel.: +46-725-941-531
}

Received: 30 November 2019; Accepted: 16 January 2020; Published: 19 January 2020

check for updates

\begin{abstract}
We present the quantum-like model of information processing by the brain's neural networks. The model does not refer to genuine quantum processes in the brain. In this model, uncertainty generated by the action potential of a neuron is represented as quantum-like superposition of the basic mental states corresponding to a neural code. Neuron's state space is described as complex Hilbert space (quantum information representation). The brain's psychological functions perform self-measurements by extracting concrete answers to questions (solutions of problems) from quantum information states. This extraction is modeled in the framework of open quantum systems theory. In this way, it is possible to proceed without appealing to the state's collapse. Dynamics of the state of psychological function $F$ is described by the quantum master equation. Its stationary states represent classical statistical mixtures of possible outputs of $F$ (decisions). This model can be used for justification of quantum-like modeling cognition and decision-making. The latter is supported by plenty of statistical data collected in cognitive psychology.
\end{abstract}

Keywords: brain; information processing; quantum-like model; action potential; uncertainty; quantum information state; neural code; neuron firing; frequency; open quantum systems; quantum master equation; differentiation

\section{Introduction}

Recent years have been characterized by tremendous development of quantum information theory and engineering. We note that from the quantum information viewpoint, it is not surprising that the quantum formalism can be used to model behavior of cognitive systems (see Reference [1,2]). And we can point to the wave of interest to quantum-like models in cognition, psychology, and decision-making. The main stimulus for development of quantum-like modeling came from decision theory that has been suffering of numerous paradoxes, some of them unresolvable by means of the traditional methods.

We point out that Tversky and Kahenman [3,4] and other researchers in psychology and economics (starting with the seminal paradoxes of Allais [5] and Ellsberg [6]) demonstrated cases where classical probability $(\mathrm{CP})$ prescription and actual human thinking persistently diverge, at least relative to baseline classical intuitions. There is a plenty of probabilistic data that does not match the laws of CP. These data were typically related to probability fallacies and irrational behavior. Tversky and Kahenman advertised the heuristic approach as an alternative to CP-modeling.

However, it was shown that some of the main problems of the CP-based decision-making can be resolved on the basis of quantum probability (QP) calculus. The QP-approach to modeling of decision-making is a purely operational approach describing probability distributions of observations' 
outputs. The quantum formalism is used to describe aforementioned data, to resolve paradoxes, and to model various psychological effects, such as conjunction, disjunction, and order effects; see, e.g., monographs $[1,2,7-10]$ and some representative papers [11-17]. The main tool is the machinery of quantum interference for incompatible observables $[1,8]$; see also $[2,18]$ for tests of contextuality in decision-making based on the Bell-type [19] inequalities. In many cases, models based on quantum theory can be seen as providing a formalization of relevant heuristic principles (e.g., the quantum model for the conjunction fallacy [12] can be thought of as a formalization of Tversky and Kahneman's representativeness heuristic [4]).

As is often the case with cognitive models [20], in the quantum-like approach, the brain is considered as $a$ black bo $x$ that processes information in accordance with the laws of quantum information theory and generates QP-data.

The quantum-like community is multi-disciplinary, with around two hundred experts (approximately 5500 links at Google scholar). The main problem of quantum-like modeling is its shaky neurophysiological ground. Although the black box model has found numerous applications, one can be curious in coupling between the quantum information model of processing of mental information by the brain and neurophysiological processes in the brain. The first steps in this direction were done in Reference [21-23].

We now briefly describe the basis components of the quantum-like model coupling electrochemical process in the brain with quantum information procession of information:

- Uncertainty generated by the action potential of a neuron is represented as quantum-like superposition of the basic mental states corresponding to a neural code (Section 2).

- The brain's psychological functions perform self-measurements by extracting from quantum information states concrete answers to questions, or solutions of problems (Section 4).

- This extraction is modeled in the framework of theory of open quantum systems (Section 6). (In this way, it is possible to proceed without appealing to the state's collapse. The latter is the most mystical postulate of quantum theory (see Section 5). Thus, we do not want to use it.)

- Dynamics of the (quantum information) state of psychological function $F$ is described by the quantum master equation (Section 6). Its stationary states represent classical statistical mixtures of possible outputs of $F$ (decisions).

- The detailed structure of approaching decision-states is modeled as the process of differentiation (Section 7). This is the novel contribution to theory of open quantum systems [16]. It provides a detailed step-by-step description of transformation of superposition to classical statistical mixture. It seems to be a promising technique that can be applied to a variety of problems in cognitive and biological modeling.

Thus, through interaction with electrochemical environment, $F$ (considered as an open system) resolves uncertainty that was originally encoded in superposition representing action potentials of neurons. This scheme can be called decision through decoherence of the mental state. This scheme was briefly described in Reference [23], and, in the this paper, we complete it with more detail, especially by analyzing different models of quantum measurement and their matching to functioning of the brain's psychological functions.

We note that one of the main distinguishing features of quantum representation is the linearity of state space. Hence, dynamics are also linear and steady states are approached exponentially quickly. So, at the quantum information level, state processing is very rapid, in contrast to the nonlinear dynamics of, say, the Hodgkin-Huxley equation [24] (see Section 3).

We underline that, in the model under consideration, mental uncertainty and its resolution are not based on genuine quantum physical processes in the brain (cf. [25-35]). Methodologically, our approach matches the ontic-epistemic structure of scientific theories (see Atmanspacher [36]). There are two level of descriptions: ontic for physical reality and epistemic for information extracted from physical reality. At the same time, the methods presented in this paper (Sections 6 and 7), based 
on theory of open quantum systems, can also be useful for genuine quantum physical modeling of the brain's functioning.

Our model is theoretical. It supported by experimental data, only indirectly, through coupling to quantum-like modeling in cognition and decision-making. And the latter has solid experimental support and plenty of statistical data that match QP but not CP (see, e.g. [1-6,8-10]. The describes the process of generation of QP-data from classical electrochemical processes in the brain.

\section{Representing Electrochemical Uncertainty in Action Potentials by Quantum-Like Superposition}

The main idea behind our model [23] connecting electrochemical processes in neural networks of the brain with the quantum-like probabilistic structure of decision-making by humans is that neurons' states can be expressed by normalized vectors of complex Hilbert space. This formalization reflects the basic feature of quantum-like information processing by neurons:

A single neuron has to 'work' with probabilistic superpositions of states carrying cognitive information.

Another crucial point is that a neural cell cannot be considered as an isolated bio-physical system. Its state of superposition is coupled with superposition states of neurons in a neural network performing some task. A neuron is treated as an open system, in which its state is affected by information flows from the electrochemical environment composed of signals (electrical currents, electromagnetic field, neurotransmitters, etc.) generated by other neurons.

We recall that the classical information approach to modeling of brain functioning explores discrete states of a neuron corresponding to various neural codes [37], e.g., 0/1, quiescent/firing neural code. We stress the role of states of uncertainty, e.g., neither quiescent nor firing, in information processing. Such states can be represented as superpositions. Our aim is to lift processing of such states to the quantum-like statistics of outputs of a psychological function $F$.

We propose to describe uncertainty in generation of an action potential by a neuron, say $\mathcal{N}$, by using states' superposition. Consider two dimensional complex Hilbert space $H$ (qubit space). At a concrete instant of time, a neuron's state can be mathematically described by a superposition of two states, quiescent and firing, labeled by $|0\rangle,|1\rangle$ :

$$
|\psi\rangle=c_{0}|0\rangle+c_{1}|1\rangle,\left|c_{0}\right|^{2}+\left|c_{1}\right|^{2}=1
$$

It is assumed that these states are orthogonal and normalized, i.e., $\langle 0 \mid 1\rangle=0$ and $\langle\alpha \mid \alpha\rangle=1, \alpha=0,1$. Here, the coordinates $c_{0}$ and $c_{1}$, with respect to the quiescent-firing basis, are complex amplitudes representing potentialities for a neuron to be quiescent or firing. Probabilistically, these potentialities are expressed by the Born rule of quantum theory:

$$
p_{\alpha}=\left|c_{\alpha}\right|^{2}=|\langle\psi \mid \alpha\rangle|^{2}
$$

We proceed with the frequency approach to probability. In the conventional classical model of probability (Kolmogorov, 1933 [38]), this approach, i.e., coupling of probability with the frequency of occurrences, is based on the law of large numbers; see also von Mises frequency theory [39]. Let $|\psi\rangle$ be a steady state, with respect to the dynamics realizing a psychological function (see Section 4). Consider a single neuron $\mathcal{N}$ and a sufficiently long time interval $T=M \Delta, M>>1$, and find the frequency $v_{M}(1)=n_{M}(1) / M$, where $n_{M}(1)$ is the number of $\Delta$-intervals such that neuron $\mathcal{N}$ produces a spike. Then, $p_{1} \approx v_{M}(1)$. We repeat that steady states play an exceptional role in our model as decision states (see Sections 6.2, 7 and works in Reference [2,15,16,23]).

An observer can count spikes. But who is the observer? In our model, the brain (more concretely, each psychological function) is a system that is able to perform self-observations: detection of information states of neurons and neural networks-but not electrochemical states, cf. with ontic-epistemic description. 
Consider now a group of neurons $G$ (say $m$ neurons) connected into a neural network. Quantum information state space of $G$ is given by tensor product of state spaces for individual neurons. If neurons do not interact, the $G$-state is factorized into tensor product of the states of individual neurons,

$$
|\psi\rangle_{G}=\left|\psi_{1}\right\rangle \otimes \ldots \otimes\left|\psi_{m}\right\rangle \equiv\left|\psi_{1} \ldots \psi_{m}\right\rangle .
$$

If neurons interact and these interactions generate correlations, their state is given by non-factorizable vector belonging to $H_{G}$, an entangled state, e.g., for two neurons,

$$
|\psi\rangle_{G}=(|00\rangle+|11\rangle) / \sqrt{2} .
$$

This state is generated by a pair of neurons firing synchronically. The coefficient $1 / \sqrt{2}$ gives the amplitude of probabilities $p(00)=p(11)=1 / 2$ (hence, for a sufficiently long time interval $T$, the proportion of periods synchronized firing and relaxation). Consider also the state

$$
|\psi\rangle_{G}=(|01\rangle+|10\rangle) / \sqrt{2}
$$

In this state, neurons fire in anti-phase; if $\mathcal{N}_{1}$ fires, then $\mathcal{N}_{2}$ does not, and vice versa.

Generally, entangled states give the quantum information representation of correlations. To illustrate this, consider a factorizable state, say,

$$
|\psi\rangle_{G}=\frac{1}{2}(|0\rangle+|1\rangle) \otimes(|0\rangle+|1\rangle)=\frac{1}{2}(|00\rangle+|01\rangle+|10\rangle+|11\rangle) .
$$

This state represents functioning of a network of two neurons such that all possible combinations of firing and relaxation are equally possible. There are no correlations in regimes of firings of $\mathcal{N}_{1}$ and $\mathcal{N}_{2}$.

So, to approach correlations in quantum information representation, neuronal networks should generate entangled states. For example, for a neural network composed of $m$ neurons, the synchronized firing generates an entangled state of the form:

$$
|\psi\rangle_{G}=c_{0}|00 \ldots 0\rangle+c_{1}|11 \ldots 1\rangle,
$$

where $\left|c_{0}\right|^{2}+\left|c_{1}\right|^{2}=1$. These coefficients give the complex amplitudes of probabilities $p(00 \ldots 0)=\left|c_{0}\right|^{2}, p(11 \ldots 1)=\left|c_{1}\right|^{2}$ (hence, for a sufficiently long time interval $T$, the proportion of periods of synchronized firing and relaxation). Synchronized firing of neurons performing realization of some psychological function seems to be the easiest way to generate correlations at the level of quantum information representation via entangled states of the form (7).

We stress that, although entangled states generated by synchronized neuronal firings are important mental resource, the quantum-like brain is not rigidly coupled to such states. A violation of synchronicity of firings does not destroy the quantum information representation. The frequency interpretation of probabilistic superpositions is still applicable. Even chaotic behavior at the neuronal level (see Section 3) generates quantum-like superpositions.

We also remark that the states considered above, Equations (3)-(7), are symmetric; neurons' permutation does change them. They belong to symmetric subspace $H_{G}^{\text {sym }}$ of state space $H_{G}$. Neurons in such states are indistinguishable. The latter seems to play the important role in computational stability. The question whether the quantum-like model of the brain's functioning should be based on symmetric state space $H_{G}^{\text {sym }}$ (reflecting indistinguishability of neurons) or on the complete quantum information state space $H_{G}$ is complex. We speculate that some psychological functions use the indistinguishability of neurons, and they process states belonging to $H_{G}^{\text {sym }}$; others work in $H_{G}$ (see also [23]). 


\section{From Nonlinear Dynamics of Electrochemical States to Linear Dynamics of Quantum-Llike Mental States}

The Hodgkin-Huxley equation [24] is the ordinary differential equation describing the dynamics of the action potential of a neuron. The dynamics are nonlinear. Nonlinearity is crucial to represent the non-periodic (chaotic) pattern of activation appearing at some physical conditions (see, for example, [40-42]). Nonlinearity is a necessary condition of chaos [43].) However, although the chaotic patterns are typical for electrochemical processes in the brain, they are not characteristic for cognitive processes. Chaotic behavior is never detected at the cognition level一at least, for psychically healthy people.

Rather, as pointed out in our model, the frequency of activations in a long interval $T$ seems to be truly important to capture cognition process. The quantity of frequency is determined regardless of chaotic or periodic behavior at the electrochemical level. Corresponding to the frequency probability, the superposition state can be assigned for a neuron. Generally, this is the superposition Equation (1). Mathematically, such states can be described by the quantum mechanical formalism based on the complex linear space representation. Quantum dynamics is linear. Thus, by elevating the electrochemical states to quantum information states, or superpositions, the brain transfers the non-linear dynamics of the Hodgkin-Huxley type into the linear quantum dynamics. The quantum-like representation is free-form chaotic patterns characteristic for underlying electrochemical representation.

\section{Quantum-Like Modeling of Functioning of Psychological Functions}

In accordance with previous consideration, a neural network $G$ generates quantum-like states, linear superpositions of the basic states $(|\alpha\rangle)$ corresponding to the neural code generated by $G$. Denote this state space by the symbol $H\left(=H_{G}\right)$.

Consider a psychological function $F$ that is physically based on neural network $G$. We suppose that $F$ has discrete outputs $\left(f_{j}\right)$ (possible "decisions"). Mathematically, a psychological function $F$ based on $G$ is represented by another orthonormal basis $(|\gamma\rangle)$ in $H$. These are basic mental states associated with this psychological function.

Each basis vector $|\gamma\rangle$ corresponds to some value $f$ of $F$. However, generally, this correspondence need not be one-to-one: a few basis vectors can correspond to the same value $f$ of psychological function $F$. In the simplest case, $F$ has only two outputs, $f= \pm 1$ (decisions "yes" /"no"). Thus, $H=H_{-} \oplus H_{+}$, where the subspaces are generated by vectors corresponding to outputs $f= \pm 1$. Generally, $H=\oplus_{f} H_{f}$.

Let $|\psi\rangle_{0}$ be the initial mental state, superposition of the neural code basis states,

$$
|\psi\rangle_{0}=\sum_{\alpha} c_{\alpha}|\alpha\rangle, \sum_{\alpha}\left|c_{\alpha}\right|^{2}=1
$$

This representation is of the electrochemical origin but of the quantum information nature.

By our mathematical model, psychological function $F$ works to transfer this superposition into a density operator $\hat{\rho}_{F}$ that is diagonal with respect to the $F$-basis:

$$
\hat{\rho}_{F}=\sum_{\gamma} \lambda_{\gamma}|\gamma\rangle\langle\gamma|
$$

where $\lambda_{\gamma} \geq 0, \sum_{\gamma} \lambda_{\gamma}=\operatorname{Tr} \hat{\rho}_{F}=1$. In quantum physics, this transition is known as decoherence. So, in our model, a psychological function generates its outputs via decoherence of the initial mental state.

In terms of density operators, $F$ generates transformation

$$
\left.\hat{\rho}_{0} \equiv|\psi\rangle_{0}\right\rangle|\psi\rangle_{0} \rightarrow \hat{\rho}_{F}
$$

This output-state can be considered as a classical state-classical with respect to psychological function $F$ (the basis $(|\gamma\rangle))$. The $\hat{\rho}_{F}$ is the classical statistical mixture of pure states $(|\gamma\rangle)$ with 
probabilistic weights $p_{\gamma}=\lambda_{\gamma}$. Thus, in our model, a psychological function resolves uncertainty presented in the initial mental state $|\psi\rangle_{0}$ by "differentiating" it (see Section 7) into weighted mixture of states $(|\gamma\rangle)$ associated with $F$. The probabilities of $F$-outputs are obtained by summation of probabilities

$$
p_{f}=\sum_{|\gamma\rangle \in H_{f}} p_{\gamma}
$$

The final step of $F$-functioning, selection of the concrete output $f$, is based on a classical random generator with the probability distribution $\left(p_{f}\right)$.

A psychological function $F$ can be considered as a self-observable. In the next sections, we present the basic quantum measurement models for the process of resolution of initial uncertainty encoded in the mental state $|\psi\rangle_{0}$.

\section{Collapse of Mental Wave Function}

We start with the presentation of the canonical quantum model for transition $|\psi\rangle_{0} \rightarrow \hat{\rho}_{F}$. It is convenient to proceed with the initial state given by density operator $\hat{\rho}_{0}$. In this model (a psychological function as a quantum observable), $F$ is represented by the Hermitian operator

$$
\hat{F}=\sum_{f} f \hat{Q}_{f}
$$

where $\hat{Q}_{f}$ is the orthogonal projector on the subspace $H_{f}$ composed of eigenvectors with the eigenvalue $f$, i.e., $\hat{Q}_{f}=\sum_{|\gamma\rangle \in H_{f}}|\gamma\rangle\langle\gamma|$.

We emphasize that mathematically a psychological function is represented by as orthonormal basis, not directly as a Hermitian operator. Realization of $F$ by $\hat{F}$ see (Equation (12)) shadows the internal structure of mental states generated by $F$. A psychological function primarily generates the special mental states, not $f$-outputs.

The value $f$ is observed with the probability

$$
p_{f}=\operatorname{Tr} \hat{\rho}_{0} \hat{\mathrm{Q}}_{\mathrm{f}}
$$

and the post-observation state with specified value $f$ is given by

$$
\hat{\rho}_{f}=\frac{\hat{Q}_{f} \hat{\rho}_{0} \hat{Q}_{f}}{\operatorname{Tr} \hat{Q}_{\mathrm{f}} \hat{\rho}_{0} \hat{\mathrm{Q}}_{\mathrm{f}}}
$$

and without output specification by the state

$$
\hat{\rho}_{F}=\sum p_{f} \hat{\rho}_{f} .
$$

In particular, for pure initial state $|\psi\rangle_{0}$ (with the density operator $\hat{\rho}_{0}=\left|\psi_{0}\right\rangle\left\langle\psi_{0}\right|$ ), the post-measurement state is always, again, the pure state:

$$
\left|\psi_{f}\right\rangle=\hat{Q}_{f}\left|\psi_{0}\right\rangle / \| \hat{Q}_{f}\left|\psi_{0}\right\rangle \|
$$

This is the famous projection postulate, often called the von Neumann projection postulate. However, von Neumann proposed [44] to use this postulate only for observables represented by Hermitian operators with non-degenerate spectra, i.e., in the case of one dimensional projectors $Q_{f}$. In the case of operators with degenerate spectra, von Neumann presented a more general scheme that has some elements of the modern theory of quantum instruments, cf. [45-47]. The general applicability of the projection postulate, i.e., irrespectively to (non-)degeneration of spectrum was postulated by Lüders [48]. Therefore, often state-transformation by Equation (16) is called the Lüders 
projection postulate. Quantum information theory is based on this postulate. The difference between the von Neumann and Lüders approaches to generation of post-measurement states also makes a difference in the basic quantum technological schemes, cryptography, computing, and teleportation (see Reference [49]).)

This mathematical model leads to jump-like state-transformation, $\left|\psi_{0}\right\rangle \rightarrow\left|\psi_{f}\right\rangle$; this transformation is often called "wave function collapse." A plenty of quantum(-like) models of cognition refer to "mental state collapse" [27,28,33-35]. However, even in genuine quantum physics the notion of wave function collapse (and generally appealing to the projection postulate [44,48]) is the most doubtful notion of quantum theory. Its straightforward use in modeling of cognition mystifies the brain's functioning.

Finally, we point to another mismatching of the canonical mathematical description of quantum measurement with our model of functioning of psychological functions. We remark that if the output value is not specified, then the initial state $|\psi\rangle_{0}$ is transferred into statistical mixture of pure states:

$$
\hat{\rho}_{F}=\sum p_{f}\left|\psi_{f}\right\rangle\left\langle\psi_{f}\right|
$$

If the correspondence $|\gamma\rangle \rightarrow f$ is not one-to-one, i.e., projectors $\hat{Q}_{f}$ need not be one dimensional, then the the $\gamma$-basis structure of the output state $\hat{\rho}_{F}$ (see Equation (9)) is washed out. And this is the good place to repeat that, in our model, a psychological function is determined on its own basis $(|\gamma\rangle)$.

\section{Open Quantum Systems Approach to Modeling of Psychological Functions}

In modeling of the brain's functioning, our aim is to proceed without mental state collapse, without referring to the projection postulate. Here, we appeal to theory of open quantum system and corresponding treatment of the measurement process $[45-47,50]$. In this theory, observation (measurement) on a system $S$ is considered as interaction of $S$ with its environment $\mathcal{E}$. The system's state dynamics generated by this interaction is described by the quantum master equation.

\subsection{Quantum Markovean Dynamics of Mental State}

Under the assumption that quantum dynamics is Markovean, this is the Gorini-KossakowskiSudarshan-Lindblad (GKSL) equation [50],

$$
\frac{d \hat{\rho}}{d t}(t)=-[\hat{\mathbf{H}}, \hat{\rho}(t)]+\hat{L} \hat{\rho}(t), \hat{\rho}(0)=\hat{\rho}_{0},
$$

where $\hat{\mathbf{H}}$ is Hamiltonian of $S$, and $\hat{L}$ is a linear operator acting in the space of linear operators (a super-operator). Commonly, operator $\hat{H}$ represents the state dynamics in the absence of outer environment. The general situation is more complicated, and operator $\hat{H}$ can contain some environmental contribution. For "natural" systems, environments, and interactions (encoded in operators $\hat{\mathbf{H}}$ and $\hat{L}$ ), the state $\hat{\rho}(t)$ asymptotically approaches some steady state $\hat{\rho}_{F}$, the solution of the stationary equation:

$$
\left[\hat{\mathbf{H}}, \hat{\rho}_{F}\right]=\hat{L} \hat{\rho}_{F} .
$$

This state is considered as the post-measurement state. The state $\hat{\rho}_{F}$ should be diagonal with respect to the $F$-basis $(|\gamma\rangle)$, i.e., it has the form (9): $\hat{\rho}_{F}=\sum_{\gamma} \lambda_{\gamma}|\gamma\rangle\langle\gamma|$. Probabilities $p_{f}$ are determined via summation, as in Equation (11).

Applications of this theory outside of genuine quantum physics were presented in the series of our previous publications $[2,15,16,23]$. In the present study, the role of $S$ is played by neural network $G$ (that can be reduced even to a single neuron), and $\mathcal{E}$ is its electrochemical environment, including electrical and chemical signals from other brain's networks, working with other psychological functions.

We stress that genuine quantum physics widely operates with isolated systems and the Schrödinger equation describes dynamics of such systems. However, an isolated biological system 
is dead (cf. [51]). Therefore, in our modeling of the brain's functioning, the notion of an isolated (even approximately) system is meaningless.

\subsection{General Quantum Dynamics of Mental State}

A system, in our case neural network $G$, and environment $\mathcal{E}$ are considered as a compound quantum(-like) system. Its state space is mathematically represented as the tensor product of state spaces of subsystems, i.e., as $\mathcal{H}=H \otimes K$, where $H=H_{G}, K=K_{\mathcal{E}}$. For a psychological function $F$, its state's dynamics is described by the Schrödinger equation,

$$
i \frac{d}{d t}=\hat{\mathbf{H}}_{F}|\Psi\rangle(t),|\Psi\rangle(0)=|\Psi\rangle_{0},
$$

i.e., $|\Psi\rangle(t)$ evolves unitary, $|\Psi\rangle(t)=U_{F}(t)|\Psi\rangle_{0}$, where $U_{F}(t)$ is one parametric group of unitary operators, $U_{F}(t): H \rightarrow H$. Here, $\hat{\mathbf{H}}_{F}$ is Hamiltonian of the compound system.

The state of $\rho(t)$ of the neural network $G$ (its quantum information state) is obtained via averaging the state $\hat{R}(t)=|\Psi(t)\rangle\langle\Psi(t)|$ of the compound system with respect to the degrees of freedom of environment $\mathcal{E}$ :

$$
\hat{\rho}(t)=\operatorname{Tr}_{K} \hat{R}(t) .
$$

We remark that the partial trace is again a pure state only for factorizable states of a compound system, i.e., generally it is a mixed state represented by a density operator. Generally, this dynamics is non-Markovean with the memory effects.

As in the Markovean case, in Section 6.1, outputs of $F$ (decisions) are generated via approaching of the steady state,

$$
\lim _{t \rightarrow \infty} \hat{\rho}(t)=\hat{\rho}_{F}
$$

which is diagonal in the F-basis $(|\gamma\rangle): \hat{\rho}_{F}=\sum_{\gamma} \lambda_{\gamma}|\gamma\rangle\langle\gamma|$. This state, or more precisely, this decomposition of the density operator, is the classical statistical mixture of the basic mental states determining the psychological function. The probabilities of $F$-outputs are given by Equation (11). The concrete value of $F$ is selected by a classical random generator with the probability distribution $\left(p_{f}\right)$.

Finally, we make a remark on the limit-procedure Equation (22). Of course, to approach the decision state $\hat{\rho}_{F}$, the network works only a finite period of time-until state's fluctuations become small with respect to stabilization parameter $\epsilon>0$. This parameter is one of characteristics of the psychological function $\epsilon \equiv \epsilon_{F}$; in principle, the model can be more complicated with $\epsilon$ depending on the initial state.

\subsection{Discussion on Open Quantum System Approach}

Appealing to the theory of open quantum systems and the use of the quantum master equation (see Equation (21) and its Markovean version Equation (18)) provide the possibility to proceed without "mental state collapse", as resulting from decision-making. However, this measurement scheme is too abstract. We do not take into account the internal structure of the process of "differentiation" of the initial state into mixture of $F$-basic states $(|\gamma\rangle)$.

We also point that stabilization to steady state $\hat{\rho}_{F}$ deforms probabilities encoded in the initial state $\rho_{0}$. Generally, the output probability $p_{\gamma}=\left\langle\gamma\left|\hat{\rho}_{F}\right| \gamma\right\rangle$ is not equal to the input probability $p_{0 \gamma}=\left\langle\gamma\left|\hat{\rho}_{0}\right| \gamma\right\rangle$. Consequently, even the probabilities for the outputs of psychological function $F$ can be modified; generally,

$$
p_{0 f}=\sum_{|\gamma\rangle \in H_{f}} p_{0, \gamma} \neq p_{f}=\sum_{|\gamma\rangle \in H_{f}} p_{\gamma}
$$


To be more concrete, consider a pure initial state $\hat{\rho}_{0}=\left|\Psi_{0}\right\rangle\left\langle\Psi_{0}\right|$. This state encodes potentiality of realization of state $|\gamma\rangle$ with the probability $p_{0 \gamma}=\left|\left\langle\Psi_{0} \mid \gamma\right\rangle\right|^{2}$. These potentialities correspond to electrochemical uncertainty generated by action potentials of neurons. It would be natural to expect that these potentialities would coincide with potentialities encoded in the output state $\hat{\rho}_{F}$ that are given by probabilities $p_{\gamma}$. However, generally $p_{\gamma} \neq\left|\left\langle\Psi_{0} \mid \gamma\right\rangle\right|^{2}$. Roughly speaking, the stabilization scheme based on the quantum master equation is too general; it generates too wide a class of the output distributions. One can desire a quantum measurement scheme without the projection postulate and with preservation of the probabilities. Such a scheme will be presented in the next section.

We remark that the canonical quantum measurement scheme based on the projection postulate (Section 5) reproduces the probability distribution of F-outputs $\left(p_{f}\right)$ encoded in the initial state $\hat{\rho}_{0}$, see Equation (24):

$$
p_{f}=\operatorname{Tr} \hat{\rho}_{0} \hat{\mathrm{Q}}_{\mathrm{f}}=\sum_{|\gamma\rangle \in \mathrm{H}_{\mathrm{f}}} \mathrm{p}_{0 \gamma} .
$$

However, the projection postulate scheme [44] suffers of the collapse-like state-transition. We want to open the "collapse back box" and to preserve probability distribution. We also repeat that the projection scheme does not describe the internal structure of projectors $\hat{Q}_{f}$, coupling to the collection of mental states $(|\gamma\rangle)$ determining the psychological function.

\subsection{Pure-State Decompositions of Density Operator as Signatures of Environments}

A density operator is commonly interpreted as a mixed state: statistical mixture of pure states. However, this interpretation is really inconsistent: density operator $\hat{\rho}$ permits different decompositions into mixtures, and statistical ensembles of pure states:

$$
\hat{\rho}=\sum q_{i}\left|\psi_{i}\right\rangle\left\langle\psi_{i}\right|, \text { where } q_{i} \geq 0, \sum_{i} q_{i}=1 .
$$

Theory of open quantum systems matches well with the following interpretation of states given by density operators. By the Naimark's dilation theorem, density operator $\hat{\rho}$ representing the state of system $S$ can be generated as a partial trace of a pure state of the larger system, $S+\mathcal{E}$. By this interpretation, it is meaningless to interpret $\hat{\rho}, S^{\prime} s$ state, intrinsically, i.e., without coupling to environment $\mathcal{E}$. Different decompositions of $\hat{\rho}$ into statistical mixtures of pure states correspond to a variety of interactions and environments. According to D'Ariano [52,53], only a pure state of system $S$ is informationally complete. A state given by a density operator that does not correspond to a pure state of $S$ is informationally incomplete: it carries the impact of environment $\mathcal{E}$; different environments can generate the same $\rho$-state.

\section{Mental State Differentiation}

We now present the model of differentiation [17] by which a system $S$ experiences step-by-step state transitions under the influence of environmental factors. This approach can be used for modeling various natural and mental phenomena: cell's differentiation, evolution of biological populations, decision-making, etc. In this paper, we apply the state-differentiation scheme to model generation of outputs of psychological function $F$ as differentiation of the initial state $\psi_{0}$ into a classical mixture of the basic states $(|\gamma\rangle$-states determining $F$. Thus, we model the process of transition of the mental state $\psi_{0}$ generated by a neural network $G$ from action potentials

Let us consider a typical state transition caused by a quantum measurement (in our model, functioning of a psychological function $F$ ) :

$$
\psi_{0} \rightarrow\left(|\gamma\rangle, p_{\gamma}\right) .
$$

Here, as in above consideration, $\psi_{0}$ denotes the initial state of $F$ represented by density operator $\hat{\rho}_{o} \equiv\left|\psi_{0}\right\rangle\left\langle\psi_{0}\right| ;\left(|\gamma\rangle, p_{\gamma}\right)$ denotes a classical statistical mixture of the basis states for $F$. 
The initial state $\psi_{0}$ can be expanded with respect to the $F$-basis as

$$
\left|\psi_{0}\right\rangle=\sum_{\gamma} \sqrt{p_{\gamma}}|\gamma\rangle
$$

where $\sqrt{p_{\gamma}}$ denotes a complex number satisfying $\left|\sqrt{p_{\gamma}}\right|^{2}=p_{\gamma}$, i.e., it contains the phase, as well. Hence, the density operator corresponding to the initial state can be expressed as the sum of two terms, diagonal and off-diagonal,

$$
\left|\psi_{0}\right\rangle\left\langle\psi_{0}\left|=\sum_{\gamma} p_{\gamma}\right| \gamma\right\rangle\left\langle\gamma\left|+\sum_{\gamma \neq \gamma^{\prime}} \sqrt{p_{\gamma}}{\sqrt{p_{\gamma^{\prime}}}}^{*}\right| \gamma\right\rangle\left\langle\gamma^{\prime}\right|
$$

The first term $\sum_{\gamma} p_{\gamma}|\gamma\rangle\langle\gamma|$ corresponds to the classical probability distribution $\left\{|\gamma\rangle, p_{\gamma}\right\}$. In physics, the process of vanishing of the second term is known as "decoherence." It represents accomplishment of the measurement of observable with $\gamma$-basis. The relation of $\psi_{0}$ and $\left\{\gamma, p_{\gamma}\right\}$ is represented as

$$
\sum_{\gamma} M_{\gamma}\left|\psi_{0}\right\rangle\left\langle\left.\psi_{0}\left|M_{\gamma}=\sum_{\gamma}\right|\left\langle\gamma \mid \psi_{0}\right\rangle\right|^{2} \mid \gamma\right\rangle\left\langle\gamma\left|=\sum_{\gamma} p_{\gamma}\right| \gamma\right\rangle\langle\gamma|,
$$

with the use of projection operator $M_{\gamma}=|\gamma\rangle\langle\gamma|$. The transition probability $\left|\left\langle\gamma \mid \psi_{0}\right\rangle\right|^{2}$ is equal to $p_{\gamma}$. Thus, in this model, the initial probability distribution encoded in $\left|\psi_{0}\right\rangle$ is not deformed. We want to realize projection transformation, as in Equation (27), as a process, i.e., to exclude the collapse-like state transformation.

We consider such a process differentiation, where the state of psychological function $F$ is differentiated to $\left\{\gamma, p_{\gamma}\right\}$ step-by-step through a large number of state transitions. Each iteration is represented by a map $\Lambda$ in the space of density operators, a "quantum channel" in the terminology of quantum information theory. State iterations are given by

$$
\hat{\rho}(0)=\left|\psi_{0}\right\rangle\left\langle\psi_{0}\right| \rightarrow \hat{\rho}(1)=\Lambda \hat{\rho}(0) \rightarrow \hat{\rho}(2)=\Lambda \hat{\rho}(1) \rightarrow \cdots \rightarrow \hat{\rho}(n)=\Lambda \hat{\rho}(n-1) .
$$

This process of iterations is called differentiation [17], if

$$
\lim _{n \rightarrow \infty} \hat{\rho}(n)=\sum_{\gamma} p_{\gamma}|\gamma\rangle\langle\gamma|
$$

is satisfied.

The concrete construction of such a channel $\Lambda$ was described in Reference [17], and now we represent it briefly by coupling with the quantum-like model of the brain's functioning. The electrochemical environment of neural network $G$ (on that psychological function $F$ is based) has many components. The initial quantum information states of these components do not depend on the initial state $\left|\psi_{0}\right\rangle$.

Let $|\Phi\rangle\langle\Phi|$ be the initial state of one of components of the environment; it belongs to environment's state space denoted by $K\left(=\mathbb{C}^{N}\right)$. The initial state of the compound system belongs to state space $H \otimes K$. We assume that it is factorized in the tensor product of the states of the subsystems:

$$
\left|\psi_{0}\right\rangle\left\langle\psi_{0}|\otimes| \Phi\right\rangle\left\langle\Phi|\equiv| \psi_{0} \Phi\right\rangle\left\langle\psi_{0} \Phi\right|
$$

In our model, already the first iteration through the channel destroys separability and generates the entangled state. Canonically, a quantum channel is realized with the aid of a unitary transformation $U$ of the state space of a compound system:

$$
U\left|\psi_{0}\right\rangle\left\langle\psi_{0}|\otimes| \Phi\right\rangle\langle\Phi| U^{*}
$$


The operator $U$ describes mathematically the correlation generated between the state of the psychological function and the environment. In Reference [17], the following concrete construction was proposed:

$$
U=\sum_{\gamma}|\gamma\rangle\langle\gamma| \otimes u_{\gamma}
$$

Here, each operator $u_{\gamma}: K \rightarrow K$ is unitary. And, more concretely,

$$
U\left|\psi_{0}\right\rangle \otimes|\Phi\rangle=\sum_{\gamma} \sqrt{p_{\gamma}}|\gamma\rangle \otimes\left|\Phi_{\gamma}\right\rangle
$$

where

$$
\left|\Phi_{\gamma}\right\rangle=u_{\gamma}|\Phi\rangle
$$

It can be shown that, if

$$
\left|\Phi_{\gamma}\right\rangle \neq\left|\Phi_{\gamma^{\prime}}\right\rangle
$$

for some $\gamma \neq \gamma^{\prime}$, then the output state is entangled, i.e., it cannot be factorized into the state of the psychological function and surrounding environment.

Take, now, in Equation (30), the spatial trace with respect to $K$. We select the orthonormal basis in $K$, say $\left(\left|\psi_{j}\right\rangle\right)_{j=1}^{N}$, and obtain that

$$
\Lambda\left(|\gamma\rangle\left\langle\gamma^{\prime}\right|\right)=\sum_{j=1}^{N}\left\langle\Phi_{\gamma} \mid \psi_{j}\right\rangle\left\langle\psi_{j} \mid \Phi_{\gamma^{\prime}}\right\rangle|\gamma\rangle\left\langle\gamma^{\prime}\left|=\left\langle\Phi_{\gamma} \mid \Phi_{\gamma^{\prime}}\right\rangle\right| \gamma\right\rangle\left\langle\gamma^{\prime}\right| .
$$

In Reference [17], it was shown that Equation (28) holds, i.e., quantum channel $\Lambda$ is a differentiation channel. It was also shown that the off-diagonal terms in density operators $\hat{\rho}(n)$ approach zero with factors $\left\langle\Phi_{\gamma} \mid \Phi_{\gamma^{\prime}}\right\rangle^{n}$. We remark that, since operators $u_{\gamma}: K \rightarrow K$ are unitary, vector $\left|\Phi_{\gamma}\right\rangle$ has the unit norm, so $\left|\left\langle\Phi_{\gamma} \mid \Phi_{\gamma^{\prime}}\right\rangle\right|<1$.

\section{Concluding Remarks}

The quantum information revolution essentially stimulated applications of quantum formalism to model cognition and decision-making. Generally, such modeling is not based on real quantum physics; the brain is considered as a black box processing information in accordance of the laws of quantum information and probability. The natural problem of coupling of the quantum-like models with the electrochemical processes in the brain arises. Following Reference [23], we proceed towards solving this problem with the two-level ("ontic-epistemic") model, with the classical electrochemical (ontic) level, and with the quantum-like (epistemic) level.

In this model, uncertainty in generation of spikes is transformed into quantum-like superposition. The main idea is that the brain is able to transfer the non-linear dynamics of the Hodgkin-Huxley type [24] into linear quantum-like dynamics. At the level of coding, this means that a classical neural code is extended to include superposition states. By moving from the nonlinear dynamics of the electrochemical processes in neural networks, the brain is able to escape chaotic behavior. The latter is characteristic for non-linear dynamical systems, including the Hodgkin-Huxley differential equation [40-42].

We realized a psychological function as a quantum-like observable. Here, we put the emphasis to the evident fact that the brain is an open system. Since the theory of open quantum systems is the most general mathematical theory of open systems, it is natural to apply it for modeling of the brain's functioning (see Reference [2,15,16,23,29-32]). We analyzed advantages and disadvantages of the canonical quantum measurement scheme based on the projection postulate, as well as the open system model based on the quantum master equation (with Markovean and non-Markovean dynamics). Finally, we presented the differentiation scheme [17]): superposition uncertainty $\rightarrow$ classical statistical mixtures of decision states. 
The use of the quantum measurement theory to model the brain's functioning leads to the fundamental foundational problem of external versus internal (self-) observations. This is a very complex foundational problem. We plan to study it in more detail in further publications.

As aforementioned, our model is indirectly supported by experimental data (from cognitive psychology, decision-making, etc.) matching the QP-description. Of course, one can dream of the direct coupling with experiments: measuring action potentials of neurons in a neural network for some brain's function, transformation of the electrochemical state into quantum information superposition, and then monitoring the quantum state-dynamics. This is a very complex project, both theoretically and experimentally. We plan to work on it in cooperation with experts in brain science.

Author Contributions: Methodology, A.K. and M.A.; Writing-original draft, A.K. All authors have read and agreed to the published version of the manuscript.

Funding: This research received no external funding.

Conflicts of Interest: The authors declare no conflict of interest.

\section{References}

1. Khrennikov, A. Ubiquitous Quantum Structure: From Psychology to Finances; Springer: Berlin/Heidelberg, Germany; New York, NY, USA, 2010.

2. Asano, M.; Khrennikov, A.; Ohya, M.; Tanaka, Y.; Yamato, I. Quantum Adaptivity in Biology: From Genetics to Cognition; Springer: Berlin/Heidelberg, Germany; New York, NY, USA, 2015.

3. Tversky, A.; Kahneman, D. Judgment under uncertainty: Heuristics and biases. Science 1974, 185, $1124-1131$. [CrossRef] [PubMed]

4. Tversky, A.; Kahneman, D. Extensional versus intuitive reasoning: The conjunction fallacy in probability judgment. Psychol. Rev. 1983, 90, 293-315. [CrossRef]

5. Allais, M. Le comportement de l'homme rationnel devant le risque: Critique des postulats et axiomes de l' cole amricaine. Econometrica 1953, 21, 503-536. [CrossRef]

6. Ellsberg, D. Risk, ambiguity and the Savage axioms. Q. J. Econ. 1961, 75, 643-669. [CrossRef]

7. Khrennikov, A. Information Dynamics in Cognitive, Psychological, Social, and Anomalous Phenomena; Kluwer: Dordreht, The Netherlands, 2004.

8. Busemeyer, J.R.; Bruza, P.D. Quantum Models of Cognition and Decision; Cambridge University Press: Cambridge, UK, 2012.

9. Haven, E.; Khrennikov, A. Quantum Social Science; Cambridge University Press: Cambridge, UK, 2013.

10. Bagarello, F. Quantum Concepts in the Social, Ecological and Biological Sciences; Cambridge University Press: Cambridge, UK, 2019.

11. Pothos, E.M.; Busemeyer, J.R. A quantum probability explanation for violation of rational decision theory. Proc. R. Soc. B 2009, 276, 2171-2178. [CrossRef] [PubMed]

12. Busemeyer, J.R.; Pothos, E.M.; Franco, R.; Trueblood, J. A quantum theoretical explanation for probability judgment errors. Psych. Rev. 2011, 118, 193-218. [CrossRef]

13. Wang, Z.; Solloway, T.; Shiffrin, R.M.; Busemeyer, J.R. Context effects produced by question orders reveal quantum nature of human judgments. Proc. Natl. Acad. Sci. USA 2014, 111, 9431-9436. [CrossRef]

14. Khrennikova, P.; Haven, E.; Khrennikov, A. An application of the theory of open quantum systems to model the dynamics of party governance in the US Political System. Int. J. Theor. Phys. 2014, 53, 1346-1360. [CrossRef]

15. Asano, M.; Basieva, I.; Khrennikov, A.; Ohya, M.; Tanaka, Y. A quantum-like model of selection behavior. J. Math. Psychol. 2017, 78, 2-12. [CrossRef]

16. Asano, M.; Basieva, I.; Khrennikov, A.; Yamato, I. A model of differentiation in quantum bioinformatics. Prog. Biophys. Mol. Biol. 2017, 130, 88-98. [CrossRef]

17. Asano, M.; Basieva, I.; Pothos, E.M.; Khrennikov, A. State entropy and differentiation phenomenon. Entropy 2018, 20, 394-404. [CrossRef]

18. Dzhafarov, E.N.; Kujala, J.V. On selective influences, marginal selectivity, and Bell/CHSH inequalities. Top. Cogn. Sci. 2014, 6, 121-128. [CrossRef] [PubMed]

19. Bell, J. Speakable and Unspeakable in Quantum Mechanics; Cambridge University Press: Cambridge, UK, 1987. 
20. Griffiths, T. L.; Chater, N.; Kemp, C.; Perfors, A.; Tenenbaum, J.B. Probabilistic models of cognition: Exploring representations and inductive biases. Trends Cogn. Sci. 2010, 14, 357-364. [CrossRef] [PubMed]

21. Khrennikov, A. Quantum-like model of processing of information in the brain based on classical electromagnetic field. Biosystems 2011, 105, 250-262. [CrossRef] [PubMed]

22. Busemeyer, J.R.; Fakhari, P.; Kvam, P. Neural implementation of operations used in quantum cognition. Progress Biophys. Mol. Biol. 2017, 130, 53-60 . [CrossRef]

23. Khrennikov, A.; Basieva, I.; Pothos, E.M.; Yamato, I. Quantum probability in decision making from quantum information representation of neuronal states. Sci. Rep. 2018, 8, 16225. [CrossRef]

24. Hodgkin, A.L.; Huxley, A.F. A quantitative description of membrane current and its application to conduction and excitation in nerve. J. Physiol. 1952, 117, 500-544. [CrossRef]

25. Umezawa, H. Advanced Field Theory: Micro, Macro and Thermal Concepts; AIP: New York, NY, USA, 1993.

26. Ricciardi, L.M.; Umezawa, H. Brain and physics of many-body problems. Kibernetik 1967, 4, 44-48. [CrossRef]

27. Penrose, R. The Emperor's New Mind; Oxford University Press: New York, NY, USA, 1989.

28. Hameroff, S. Quantum coherence in microtubules. A neural basis for emergent consciousness? J. Cons. Stud. 1994, 1, 91-118.

29. Vitiello, G. Dissipation and memory capacity in the quantum brain model. Int. J. Mod. Phys. B 1995, 9, 973. [CrossRef]

30. Vitiello, G. My Double Unveiled: The Dissipative Quantum Model of Brain; Advances in Consciousness Research; John Benjamins Publishing Company: Amsterdam, The Netherlands, 2001.

31. Sabbadini, S.A.; Vitiello, G. Entanglement and phase-mediated correlations in quantum field theory. Application to brain-mind states. Appl. Sci. 2019, 9, 3203. [CrossRef]

32. Tressoldi, P.; Pederzoli, L.; Matteoli, M.; Prati, E.; Kruth, J. Can our minds emit light at $7300 \mathrm{~km}$ distance? A pre-registered confirmatory experiment of mental entanglement with a photomultiplier. NeuroQuantology 2016, 14, 447-455. [CrossRef]

33. Bernroider, G.; Summhammer, J. Can quantum entanglement between ion transition states effect action potential initiation? Cogn. Comput. 2012, 4, 29-37. [CrossRef]

34. Bernroider, G. Neuroecology: Modeling neural systems and environments, from the quantum to the classical level and the question of consciousness. J. Adv. Neurosc. Res. 2017, 4, 1-9. [CrossRef]

35. Igamberdiev, A.U.; Shklovskiy-Kordi, N.E. The quantum basis of spatiotemporality in perception and consciousness. Prog. Biophys. Mol. Biol. A 2017, 130, 15-25. [CrossRef] [PubMed]

36. Atmanspacher, H. Determinism is ontic, determinability is epistemic. In Between Chance and Choice: Interdisciplinary Perspectives on Determinism; Atmanspacher, H., Bishop, R.C., Eds.; Imprint Academic: Thorverton, UK, 2002; pp. 49-74.

37. Brown, E.N.; Kass, R.E.; Mitra, P.P. Multiple neural spike train data analysis: State-of-the-art and future challenges. Nat. Neurosci. 2004, 7, 456-461. [CrossRef]

38. Kolmolgoroff, A.N. Grundbegriffe der Wahrscheinlichkeitsrechnung; Springer: Berlin, Germany, 1933.

39. Von Mises, R. Probability, Statistics and Truth; Macmillan: London, UK, 1957.

40. Aihara, K. Chaos in Neural Response and Dynamical Neural Network Models: Toward a New Generation of Analog Computing in Towards the Harnessing of Chaos; Yamaguti, M., Ed.; Elsevier, Science Publishers B.V. : Amsterdam, The Netherlands, 1994; pp. 83-98.

41. Aihara, K. Chaos in Axons, in The Handbook of Brain Theory and Neural Networks, 1st ed.; Arbib, M.A., Ed.; The MIT Press: Cambridge, MA, USA, 1995; pp.183-185.

42. Guckenheimer, J.; Oliva, R.A. Chaos in the Hodgkin-HuXley model. SIAM J. Appl. Dyn. Syst. 2002, 1, $105-114$. [CrossRef]

43. Timberlake T.K.; Mixon J.W. Nonlinearity and chaos. In Classical Mechanics with Maxima; Undergraduate Lecture Notes in Physics; Springer: New York, NY, USA, 2016.

44. Von Neumann, J. Mathematical Foundations of Quantum Mechanics; Princeton University Press: Princenton, NJ, USA, 1955.

45. Davies, E.B. Quantum Theory of Open Systems; Academic Press: London, UK, 1976.

46. Ozawa, M. Quantum perfect correlations. Ann. Phys. 2006, 321, 744-769. [CrossRef]

47. Ozawa, M. Probabilistic interpretation of quantum theory. N. Gen. Comput. 2016, 34, 125-152. [CrossRef]

48. Lüders, G. Uber die Zustansanderung durch den Messprozess. Ann. Phys. Lpz. 1951, 8, 322.

49. Khrennikov, A. Two versions of the projection postulate: From EPR argument to one-way quantum computing and teleportation. Adv. Math. Phys. 2010, 2010, 945460. [CrossRef] 
50. Ingarden, R.S.; Kossakowski, A.; Ohya, M. Information Dynamics and Open Systems: Classical and Quantum Approach; Fundamental Theories of Physics (Book 86); Springer: Berlin/Heidelberg, Germany, 1997.

51. Schrödinger, E. What is Life? The Physical Aspect of the Living Cell; Cambridge University Press: Cambridge, UK, 1944.

52. D' Ariano, G.M. Operational axioms for quantum mechanics. In Foundations of Probability and Physics-3; AIP Conference Proceedings; Adenier, G., Fuchs, C., Khrenniko, A., Eds.; AIP: College Park, MD, USA, 2007; Volume 889, pp. 79-105.

53. D'Ariano, G.M. Physics as information processing. In Advances in Quantum Theory; AIP Conference Proceedings; AIP: College Park, MD, USA, 2011; Volume 1327, pp. 7-16.

(C) 2020 by the authors. Licensee MDPI, Basel, Switzerland. This article is an open access article distributed under the terms and conditions of the Creative Commons Attribution (CC BY) license (http:/ / creativecommons.org/licenses/by/4.0/). 